\title{
ON COMPACT AND BOUNDING HOLOMORPHIC MAPPINGS
}

\author{
MIKAEL LINDSTRÖM
}

(Communicated by William J. Davis)

\begin{abstract}
Let $E$ and $F$ be complex Banach spaces. We say that a holomorphic mapping $f$ from $E$ into $F$ is compact respectively bounding if $f$ maps some neighbourhood of every point of $E$ into a relatively compact respectively bounding subset of $F$. Recall that a subset of $E$ is bounding if it is mapped onto a bounded set by every complex valued holomorphic mapping on $E$. Compact holomorphic mappings have been studied by R. Aron and $\mathrm{M}$. Schottenloher in [1]. Since every relatively compact subset of a Banach space is trivially bounding it is clear that every compact holomorphic mapping is bounding. We show that the product of three bounding holomorphic mappings is compact.
\end{abstract}

In this note we deal with compact and bounding holomorphic mappings on Banach spaces. We prove that the product of three bounding holomorphic mappings is compact. This result is a holomorphic analogue of a result in [6] from which follows that a locally convex space $E$ is Schwartz iff it is quasi-normable and every equicontinuous, weak*-null sequence in the dual of $E$ converges to zero in the strong topology $E_{\beta}^{\prime}$. Finally, we briefly consider the problem under which condition every holomorphic mapping from $C(K)$ into $E$ is compact, where $K$ is a compact Hausdorff space and $C(K)$ is the Banach space of continuous functions on $K$.

For Banach spaces $E$ and $F$ let $H(E, F)$ denote the vector space of all holomorphic mappings from $E$ into $F$, i.e. all continuous $F$-valued mappings on $E$ which are Gateaux-analytic. A subset $B$ of a Banach space $E$ is called limited, if every $\sigma\left(E^{\prime}, E\right)$-null sequence in $E^{\prime}$ converges uniformly to zero on $B$ and bounding, if every $f \in H(E)$ is bounded on $B$. Since a sequence $l_{n}$ in $E^{\prime}$ is $\sigma\left(E^{\prime}, E\right)$-null iff $\sum_{n=0}^{\infty} l_{n}^{n}$ is a holomorphic mapping on $E$ [4], it is easily seen that

(1) Every bounding subset of a Banach space is limited.

Further since every holomorphic mapping is continuous, we have

(2) Every relatively compact subset of a Banach space is bounding.

Received by the editors December 1, 1987 and, in revised form, March 22, 1988.

1980 Mathematics Subject Classification (1985 Revision). Primary 46G20; Secondary 46E10. 
Let us also recall that a subset $B$ of a Banach space is called conditionally weakly compact, if every sequence in $B$ contains a weak Cauchy subsequence. The following results follow from [2] because of (1) (cf. also [4]).

(3) Every bounding subset of a Banach space is conditionally weakly compact.

(4) Every bounding subset of a reflexive Banach space is relatively compact.

(5) Every bounding subset of a Banach space not containing $l_{1}$ is relatively weakly compact.

It is not known if there exists any limited set which is not bounding. For the Banach space $l_{\infty}(\Gamma), \Gamma$ arbitrary, B. Josefson [5] has proved that limited and bounding sets are the same. This is also true for every injective Banach space, because every Banach space.embeds in $l_{\infty}(\Gamma)$ for suitable $\Gamma$. When $E$ is a Grothendieck space with the Dunford-Pettis property then the limited and conditionally weakly compact subsets of $E$ coincide. Hence the three concepts limited, bounding and conditionally weakly compact coincide, if $E$ is an injective Banach space. As an example let us mention that $C(K)$ spaces are injective for compact extremally disconnected spaces $K$.

Let us now define what we mean by a compact respectively bounding holomorphic mapping.

Definition. Let $E$ and $F$ be Banach spaces. A mapping $f \in H(E, F)$ is said to be compact, weakly compact, bounding respectively Rosenthal, if for each $x \in E$ there is a neighbourhood $V_{x}$ of $x$ such that $f\left(V_{x}\right)$ is a relatively compact, relatively weakly compact, bounding respectively conditionally weakly compact subset of $F$.

Of course every compact holomorphic mapping is bounding and this in turn is Rosenthal. If the target space is an injective Banach space, then conversely every Rosenthal holomorphic mapping is bounding. Note also that a continuous $n$-homogeneous polynomial $p: E \rightarrow F$ is compact, weakly compact respectively Rosenthal, if it maps any bounded subset of $E$ to a relatively compact, relatively weakly compact respectively conditionally weakly compact subset of $F$.

In the sequel we shall need that the closed absolutely convex hull of a conditionally weakly compact set is conditionally weakly compact. This can be proved in the following way using limited sets, as was shown to the author by Thomas Schlumprecht. Indeed, let $A \subset E$ be conditionally weakly compact. Since the closed absolutely convex hull of a limited set is trivially limited, it follows that $\bar{\Gamma} A$ is limited in $l_{\infty}(\Gamma)$ for suitable $\Gamma$. Hence $\bar{\Gamma} A$ is conditionally weakly compact in $l_{\infty}(\Gamma)$, and we conclude that $\bar{\Gamma} A$ is conditionally weakly compact in $E$.

We shall now prove that a holomorphic mapping $f$ is Rosenthal, if it is Rosenthal near the origin and also that conditional weak compactness of $f$ is equivalent to conditional weak compactness of its derivatives at each point. Analogous results for compact and weakly compact holomorphic mappings have been proved in [1] and [9] respectively. We need the following lemma from [9], 
where a subset $U$ of $E$ is called circled if $\lambda x \in U$ for every $x \in U$ and every $\lambda \in C$ with $|\lambda|=1$.

Lemma 1. Let $f \in H(E, F)$. If $U \subset E$ is circled and $x \in E$, then

$$
1 / n ! \hat{d}^{n} f(x)(U) \subset \bar{\Gamma} f(x+U)
$$

for every $n \in N$.

Proposition 2. Let $f \in H(E, F)$. The following are equivalent:

(i) $f$ is Rosenthal.

(ii) $f$ maps some zero-neighbourhood in $E$ into a conditionally weakly compact subset of $F$.

(iii) $\hat{d}^{n} f(x)$ is a Rosenthal polynomial for every $n \in N$ and every $x \in E$.

(iv) $\hat{d}^{n} f(0)$ is a Rosenthal polynomial for every $n \in N$.

Proof. Because of Lemma 1 we only have to show that (iv) implies (i). Let $x \in$ $E$. Since $f \in H(E, F)$, we have for some $\varepsilon>0$ that $f(y)=$ $\sum_{n=0}^{\infty} 1 / n ! \hat{d}^{n} f(0)(y)$ uniformly for $y \in B(x, \varepsilon)$. By the assumption, for each $k \in N$, the set

$$
\left\{\sum_{n=0}^{k} 1 / n ! \hat{d}^{n} f(0)(y): y \in B(x, \varepsilon)\right\}
$$

is conditionally weakly compact. We claim that $f(B(x, \varepsilon))$ is conditionally weakly compact. Let $\left(y_{m}\right)$ be a sequence in $B(x, \varepsilon)$. We must exhibit a weak Cauchy subsequence of $\left(f\left(y_{m}\right)\right)$. Put $z_{m}^{k}=\sum_{n=0}^{k} 1 / n ! \hat{d}^{n} f(0)\left(y_{m}\right)$. Hence by a diagonal process there is a weak Cauchy subsequence $\left(z_{m_{i}}^{k}\right)$ for each $k \in N$. Then $\left(f\left(y_{m_{i}}\right)\right)$ is a weak Cauchy subsequence. Indeed, for given $l \in F^{\prime}$ and $\delta>0$, there is $k \in N$ such that

$$
\left\|f(y)-\sum_{n=0}^{k} 1 / n ! \hat{d}^{n} f(0)(y)\right\|<(3 \cdot\|l\|)^{-1} \cdot \delta,
$$

for all $y \in B(x, \varepsilon)$. Choose $n_{0} \in N$ such that for $i, j \geq n_{0}$,

$$
\left|l\left(z_{m_{i}}^{k}\right)-l\left(z_{m_{j}}^{k}\right)\right|<\delta / 3
$$

Then for $i, j \geq n_{0}$,

$$
\begin{aligned}
\left|l\left(f\left(y_{m_{i}}\right)\right)-l\left(f\left(y_{m_{j}}\right)\right)\right| \leq & \|l\| \cdot\left\|f\left(y_{m_{i}}\right)-z_{m_{i}}^{k}\right\|+\left|l\left(z_{m_{i}}^{k}\right)-l\left(z_{m_{j}}^{k}\right)\right| \\
& +\|l\| \cdot\left\|f\left(y_{m_{j}}\right)-z_{m_{j}}^{k}\right\|<\delta,
\end{aligned}
$$

and the proof is complete.

In order to prove the announced result we need a generalization to holomorphic mappings of the factorization theorem of W. Davis et al. [3]. But this has already been done by R. Aron and M. Schottenloher in [1] where they obtained a factorization theorem of compact holomorphic mappings. In [9] R. Ryan has 
used their methods to prove a factorization theorem for weakly compact holomorphic mappings. Since we need factorization of compact, weakly compact and Rosenthal holomorphic mappings we only have to consider the case when the holomorphic mappings are Rosenthal.

Proposition 3. Let $f \in H(E, F)$. Then $f$ is compact, weakly compact respectively Rosenthal iff there is a closed absolutely convex subset $L$ of $F$ which is compact, weakly compact respectively conditionally weakly compact and such that $f: E \rightarrow F_{L}$ is holomorphic, where $F_{L}=$ span $L$ normed by the Minkowski functional of $L$.

Proof. We consider the case when $f \in H(E, F)$ is Rosenthal. For each $M \in \mathbf{N}$ and $x \in E$, define

$$
A_{M}(x)=\left\{\lambda y: y \in B\left(x, M^{-1}\right),|\lambda| \leq 2\right\}
$$

and let

$$
U_{M}=\bigcup\left\{B\left(x, M^{-1}\right):\|x\| \leq M \text { and } \sup \left\{\|f(y)\|: y \in A_{M}(x)\right\} \leq M\right\} .
$$

For every $x \in E$ there is a $M \in N$ such that $\sup \left\{\|f(y)\|: y \in A_{M}(x)\right\} \leq M$ which shows that $E=\bigcup_{M \in \mathrm{N}} U_{M}$. We now show that $f\left(U_{M}\right)$ is conditionally weakly compact. Let $y \in U_{M}$ with $y \in B\left(x, M^{-1}\right)$. Then for each $n \in \mathbf{N}$

$$
\begin{aligned}
\left\|1 / n ! \hat{d}^{n} f(0)(y)\right\| & \leq 2^{-n} \sup \{\|f(\lambda y)\|:|\lambda|=2\} \\
& \leq 2^{-n} \sup \left\{\|f(y)\|: y \in A_{M}(x)\right\} \\
& \leq 2^{-n} \cdot M .
\end{aligned}
$$

Because $U_{M}$ is bounded, $\left\{\sum_{n=0}^{k} 1 / n ! \hat{d}^{n} f(0)(y): y \in U_{M}\right\}$ is conditionally weakly compact for each $k \in N$. Therefore, arguing as in the proof of Proposition 2 , we conclude that $f\left(U_{M}\right)$ is conditionally weakly compact. Now it is easily seen that

$$
K=\{0\} \cup \bigcup_{M \in \mathbf{N}}\left\{\left[M \cdot \sup \left\{\|f(y)\|: y \in U_{M}\right\}\right]^{-1} \cdot f\left(U_{M}\right)\right\},
$$

is a conditionally weakly compact set whose span contains $f(E)$. Let $L$ denote the closed absolutely convex hull of $K$. Then it can be shown as in [1, Proposition 3.5] that $f: E \rightarrow F_{L}$ is holomorphic.

Since every weakly compact linear mapping can be factorized through a reflexive Banach space and every Rosenthal linear mapping (i.e. the image of the unit ball is conditionally weakly compact) through a Banach space not containing $l_{1}$ the following corollary follows:

Corollary 3.1. Let $f \in H(E, F)$. Then $f$ is weakly compact respectively Rosenthal iff there exist a Banach space $G$ which is reflexive respectively does not contain a copy of $l_{1}$, a continuous linear mapping $g: G \rightarrow F$ and a holomorphic mapping $h: E \rightarrow G$ such that $f=g \circ h$.

Now we are ready to prove the main result. 
Theorem 4. The compact holomorphic mappings are exactly those which can be represented as a product of three bounding holomorphic mappings.

Proof. Let $E, F, G, H$ be Banach spaces and let $f \in H(E, F), g \in$ $H(F, G), h \in H(G, H)$ be bounding holomorphic mappings. Consider the following diagram

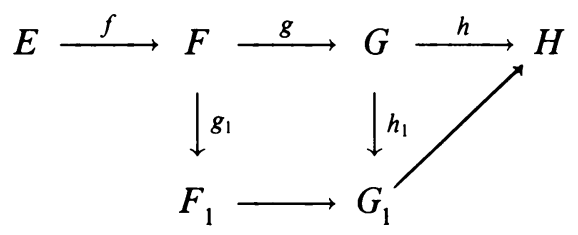

The mapping $h$ is Rosenthal and consequently $G_{1}$ does not contain $l_{1}$ according to Corollary 3.1. Hence $h_{1} \circ g \in H\left(F, G_{1}\right)$ is weakly compact by (5) and the fact that the holomorphic image of a bounding set is bounding. From Corollary 3.1, follows that $F_{1}$ is reflexive. This means that $g_{1} \circ f \in H\left(E, F_{1}\right)$ is compact, according to (4). Thus $h \circ g \circ f \in H(E, H)$ is compact, and the first part is proved. The converse implication follows from Proposition 3 and the fact that every compact linear mapping can be represented as a product of three compact linear mappings.

Since every bounding subset of a Banach space is limited, it follows that every bounding linear mapping is limited, i.e. the image of the unit ball is limited. I do not know if the converse result is true, but the main result in [6] says that the product of three limited linear mappings is compact. The abovementioned generalization of the Josefson-Nissenzweig theorem was obtained from this result. In connection with Theorem 4 we also want to point out that there is no complete characterization of those Banach spaces in which bounding subsets agree with relatively compact subsets.

We shall conclude this note by investigating when holomorphic mappings from $C(K)$ into $E$ are compact, where $K$ is a compact Hausdorff space. The proof of our remark is based on the following result due to A. Pełczyński [8]: Every continuous polynomial mapping on $C(K)$ into a Banach space $E$, which does not have a subspace isomorphic to $c_{0}$, is compact iff $K$ is a dispersed compact Hausdorff space. We denote the space of all compact holomorphic mappings from $E$ into $F$ by $H_{K}(E, F)$. The space of all compact (respectively weakly compact) linear mappings from $E$ into $F$ is denoted by $K(E, F)$ (respectively $W(E, F)$ ).

Remark. Let $K$ be a dispersed compact Hausdorff space, $C(K)$ an infinitedimensional Grothendieck space and $E$ a Banach space. If

$$
K(C(K), E)=W(C(K), E),
$$

then $H(C(K), E)=H_{K}(C(K), E)$.

Proof. . Let us assume that $E$ contains a copy of $c_{0}$. Let now $x_{n}^{\prime}$ be a $\sigma\left(C(K)^{\prime}, C(K)\right)$-null sequence in $C(K)^{\prime}$. Then the continuous linear mapping 
$T: C(K) \rightarrow c_{0}, x \mapsto x_{n}^{\prime}(x)$, is weakly compact, since $C(K)$ is a Grothendieck space. This means that the composition $C(K) \rightarrow c_{0} \hookrightarrow E$ is compact by the assumption. Hence $T$ is compact, and it follows that $x_{n}^{\prime}$ is norm null in $C(K)^{\prime}$. This is a contradiction to the Josefson-Nissenzweig theorem, and consequently we can assume that $E$ contains no copy of $c_{0}$. Take an arbitrary $f \in H(C(K), E)$. Then $\hat{d}^{n} f(0) / n !: C(K) \rightarrow E$ is compact for each $n \in N$ by the above result of A. Pelczyński. Thus $f \in H_{K}(C(K), E)$ by Proposition 3.4 in [1].

In [1] R. Aron and M. Schottenloher obtained a similar remark also based on the same result of A. Petczyński. In the case that the target space is $c_{0}$ we have the following result from [7]: Let $E$ be a quasi-normable space. Then $E$ is Schwartz iff $L\left(E, c_{0}\right)=K\left(E, c_{0}\right)$ iff $H\left(E, c_{0}\right)=H_{K}\left(E, c_{0}\right)$.

\section{ACKNOWLEDGMENT}

The author is grateful to the referee for comments and suggestions.

\section{REFERENCES}

1. R. M. Aron and M. Schottenloher, Compact holomorphic mappings on Banach spaces and the approximation property, J. Funct. Anal. 21 (1976), 7-30.

2. J. Bourgain and J. Diestel, Limited operators and strict cosingularity, Math. Nachr. 119 (1984), 55-58.

3. W. J. Davis, T. Figiel, W. B. Johnson and A. Pelczyński, Factoring weakly compact operators, J. Funct. Anal. 17 (1974), 311-327.

4. S. Dineen, Complex analysis in locally convex spaces, North Holland 1981.

5. B. Josefson, Bounding subsets of $l^{\infty}(A)$, J. Math. Pures et Appl. 57 (1978), 397-421.

6. M. Lindström, A characterization of Schwartz spaces, Math. Z. 198 (1988), 423-430.

7. __ Schwartz spaces and compact holomorphic mappings, Manuscripta Math. 60 (1988), 139-144.

8. A. Petczyński, A theorem of Dunford-Pettis type for polynomial operators, Bull. Acad. Pol. Sci. 11 (1963), 379-386.

9. R. A. Ryan, Weakly compact holomorphic mappings on Banach spaces, Pacific J. Math. 131 (1988), 179-190.

Department of Mathematics, Abo Akademi, SF-20500 Abo, Finland 